\title{
SimulaCRES ET EFFET DE RÉEl DANS UN ROMAN DE ClAUde Simon
}

\author{
Gilles GUIGUES \\ Université d'Aix-Marseille I
}

\begin{abstract}
En): By fragmenting the linearity of narration and by the permanent addition of many details, Claude Simon's duplicity with the truth of the story is used for bringing alive the meaning all around several possibilities. From time to time, in the narration, the punctilious flux of the description is stopped; then, by a kind of turning back on itself, the description tries to correct its expression to show the complex nature of things in a reality effect. In this way, such a writing of the sensation develops a world and, simultaneously, pretends to discover it in the course of the text being built.
\end{abstract}

Keywords (En): duplicity ; description ; factual ; reality effect ; perception

Mots-clés (Fr) : ruse ; description ; factuel ; effet de réel ; perception

\section{Introduction}

Le Nouveau Roman a cherché à prendre ses distances avec les conventions romanesques qui prévalaient jusqu'alors: la référence claire et précise à une intrigue portée par une trame narrative aisément repérable, l'organisation formelle autour de personnages clés, le rôle logique endossé par un narrateur unique, bref tout un lot de cadres somme toute normatifs et contraignants pour le contenu et la structuration même du roman (JANVIER : 1964). Or, en soumettant le déroulement narratif à de tels effets de brouillages, comme autant de confusions imposées au récit pour en rendre la perception toujours floue et indécise, et par conséquent en augmenter la difficulté de compréhension, les principaux acteurs de ce mouvement littéraire n'ont pas eu seulement pour finalités dernières de renouveler le genre du roman traditionnel; ils souhaitaient surtout conserver toute la part d'incertitude qui reste attachée au texte, dont la véritable nature semble se défaire de son élaboration formelle. Ici, il s'agira de s'interroger sur la fonction de l'illusion descriptive et les répercussions de tout ordre qu'elle entraîne à sa suite dans ses nombreuses interférences sur le fil narratif du récit chez Claude Simon, un de ses représentants - même si ce dernier, opposé à la rigidité de la critique qui réduit trop étroitement et uniformise à l'excès ce mouvement, préfère l'appeler «École du regard » (POIRSON et GouX, 1977 : 42).

Dans un style caractéristique, le récit s'appuie souvent plus que de mesure sur de longues descriptions fournies et minutieuses, où il parait avoir été libéré des contraintes habituelles, formelles, soumis en cela aux nombreux aléas qui en modifient la logique, comme si la narration obéissait à un principe d'incertitude permanent, défiant toute recherche de cohérence. Dans bien des romans qui peuvent en témoigner, le texte semble s'initier sous nos yeux à partir d'une existence concrète analysée dans ses moindres aspects ou encore d'un fait historique détaillé de la manière la plus objective qui soit, tout en y mêlant de manière indistincte l'invention la plus pure. Un tel procédé cherche à rendre 
indécidable la part qui relève du désir imaginaire du narrateur de celle qui concerne plus prosaïquement les données factuelles de la réalité. Ainsi pris à partie par un récit qui l'invite à le suivre dans ses errances, ses doutes, le lecteur est d'abord mobilisé et introduit dans la scène évoquée puis contraint de coopérer pour cerner au plus près l'objet même de la perception, voire la nature profonde des choses décrites - ce qui contribue à «la tonalité mythique des textes simoniens » (VAREILLE, 1989: 90). Car, en tout état de cause, c'est bien la focalisation sur la perception de la réalité qui semble occuper une telle écriture de la sensation, aiguisée par un certain regard scénarisant en permanence des effets de réel et de déréalisation jusqu'à parvenir à rendre indécidable la part empruntée à la vérité des choses de celle puisée au registre du fantasme. Voilà pourquoi cette étude a voulu analyser les capacités génératrices du récit simonien. La stratégie narrative tisse une trame complexe où le récit, en métissant le vrai et le faux, semble jouer en permanence avec la réalité qu'il cherche à approcher faute de pouvoir parfaitement la circonscrire. Il s'agira de s'appuyer surtout sur le roman Histoire, dans lequel le récit, porté par une description dynamique, semble partagé entre une certaine aliénation des faits, qu'il s'agit de restituer au mieux car ils se sont effectivement produits, et tout le possible qu'ils recèlent.

\section{Les simulacres du récit}

Très souvent, l'écriture simonienne produit une ressemblance avec un référent préexistant ; parfois, elle imite un référent qui a dû l'être, voire qui est seulement censé avoir existé. Cela amène le lecteur à établir un lien logique et indubitable entre les termes et les choses évoquées ou, au contraire, à imaginer un rapport possible entre le langage choisi et le réel à dire. La trame narrative repose toujours sur de longues descriptions qui, malgré leur densité, ne semblent pas parvenir à circonscrire parfaitement ce qu'elles cherchent à approcher, soumises aux aléas d'un récit libéré de toute certitude. À la recherche d'un sens inaccessible, tout au long d'interminables et sinueux développements, qui prennent appui sur un nombre considérable de détails précisant la scène scrutée attentivement, le récit garde ainsi un caractère incertain. Fournie, la description ne met jamais un terme au questionnement du narrateur, mais le propose tout au contraire à autant de pistes successives qui s'ouvrent au fil de son incessante avancée. L'impression demeure que, malgré les nombreuses précisions apportées, rien n'assure la fiabilité de la scène, comme si le récit ne pouvait s'appuyer sur rien de vraiment concret pour le garantir. Décrites avec le plus grand soin dans une claire volonté de ne rien omettre, les choses passées plusieurs fois sous l'éclairage d'une longue analyse conservent une incertitude impossible à lever, qui se répercute sur le sens entaché à son tour de relativité. Le sens reste problématique en dépit de tout ce qui, dans la minutie de l'examen, fait mine d'en dévoiler l'énigme, car la description ne recherche pas une forme de vérité qui authentifierait le récit. Elle porte au contraire la marque de ce à quoi elle s'affronte : la nature improbable des choses ; n'ayant rien de commun avec la vérification, prise au sens de l'empirisme logique, elle défie toute justification. Dégagé de toute certitude, le récit s'offre à une lecture active qui peut librement se loger dans l'écart jamais comblé, entre la proposition 
originaire et son interprétation. En voilant le sens véhiculé par le récit, l'intention semble vouloir montrer la faille irréductible entre la réalité et son avènement dans le texte. Et les moments de clarté dans la description, lorsque les faits exprimés et leur signification coïncident parfaitement, ont pour finalité de ne jamais décourager le lecteur. Dans ces moments de répit, où le sens paraît limpide, l'auteur s'assure un compagnonnage fidèle. Mais ces indices éclairants sont aussitôt suivis d'une phrase qui réclame une imagination active. En fragmentant la linéarité du récit par l'ajout permanent de nombreuses précisions, le sens est sans cesse relancé vers plusieurs pistes possibles. Inlassablement, la description pointilleuse à l'envi fait mine de s'avancer, puis, par une sorte de retour sur ellemême, se reprend comme pour se corriger et complexifier davantage l'écriture du réel en train de s'élaborer. Un tel effet se répercute sur la trame du récit et instille ainsi une musique lancinante, qui témoigne de l'impossibilité du savoir absolu sur toute chose. La description fait subir une transformation au fait qu'elle s'évertue à établir tout en faisant douter de son existence même. L'incertitude naît aussi de la nature même de l'écriture: pensées et sensations se mêlent au déroulement signifiant du récit, ce qui a pour effet de ménager un doute permanent. Dans sa lente progression, le texte se nourrit d'images qui le gonflent de pulsations, jusqu'à lui conférer un statut incertain de récit filmique en train de se constituer. En raison d'une ponctuation fluctuante et d'une syntaxe libre, il est parfois difficile de savoir si les phrases sont des propositions indépendantes ou bien une seule phrase qui s'étire à l'infini. Malgré une description dense et généreuse, qui semble chercher à ne rien dissimuler de la réalité, des phrases défiant toute logique ne semblent pas parfaitement ajustées entre elles et produisent par leur enchaînement une parfaite indétermination. L'écriture, soumise à une sorte d'arythmie, confère une musicalité toute particulière au texte qui semble construit à grand renfort d'approximations, de doutes accentués par de fréquentes retouches, comme autant d'aveux d'incertitude voire d'inachèvement qui constituent la matière meuble, malléable, du développement. À titre de preuve, l'usage très fréquent du participe présent qui, utilisé de manière incongrue, maintient la sensation d'une double temporalité : il laisse croire que l'action se déroule, alors que l'action, déjà écoulée, est décrite sous un autre éclairage. Dans Histoire, le participe présent est essaimé dans la phrase où se conjuguent plusieurs temporalités, mais aussi le fait objectif et le fantasme (SIMON, 1967 : 18-19) :

Il existait dans un quelque part où elle irait un jour le rejoindre un au-delà paradisiaque et vaguement oriental quelque Éden quelque jardin à l'inimaginable végétation tout bruissant du cliquetis des palmes balancées comme celles qu'elle pouvait voir ornant les timbres de ces cartes postales qu'il lui envoyait ne portant le plus souvent au verso dans la partie réservée à la correspondance qu'une simple signature au-dessous d'un nom de ville et d'une date par exemple $[\ldots]$.

Mais le récit emprunte d'autres simulacres par l'emploi fréquent de séquences du genre : comme une sorte de, ressemblait à quelque chose comme, comme pour ainsi dire, on aurait dit comme, comme si, une espèce de. Ces opérateurs permettent d'évoquer une ressemblance subjectivement ressentie, alors que la description cherche à être plus précise en usant d'un style intarissable, à catalyse 
dirait Barthes (à catalyse : dont les corrections sont des ajouts). Cet effet obsédant vise à cerner l'impression la plus juste par de multiples et d'incessants ajouts, qui, par contiguïté ou aimantation verbale, marquent à la fois une comparaison énonçant un rapport entre deux éléments et une recherche de la forme modale adéquate pour accentuer la «matérialité du signe linguistique (RICARDOU, 1978 : 185-186). Soumis à de telles modalités, le déroulement du récit est ouvert à toutes les conjectures qui le relancent par de nombreux recoupements successifs, sans vraiment parvenir à cerner son objet avec une assurance définitive; par le caractère incertain du sens qu'elle développe, la comparaison réclame de manière permanente la collaboration imaginative du lecteur. Cela suggère que la description ne peut se suffire à elle-même pour cerner la nature complexe de l'objet d'attention ni même reformer toutes ses qualités. D'où l'utilisation constante des parenthèses qui semble tout à la fois vouloir sédimenter les sensations et rendre plus précise l'appréhension de la chose décrite en complexifiant la trame du récit pour en repousser à l'infini les limites. Dans Histoire, la phrase suivante, composée d'une immense parenthèse qui en abrite deux autres, l'illustre (SIMON, $1967:$ 19-20) :

[...] et au recto (quand elle - la jeune fille qu'elle avait été - avait lu le nom de la ville la date la signature et qu'elle retournait la carte, elle et grand-mère assises l'une en face de l'autre devant leurs minuscules tasses de ce chocolat à l'espagnole qui leur détraquait le foie, si épais (recommandait-elle aux domestiques) que la petite cuiller d'argent devait rester toute droite sans s'incliner ni tomber sur le bord lorsqu'on la plantait dedans - ou encore, l'été (la carte de Colombo datée d'août avait dû l'atteindre alors que comme chaque année elles étaient déjà parties s'installer à la propriété) dans le jardin étincelant, vêtue d'un de ces flasques et austères peignoirs à collerette boutonnée jusqu'au cou, aux pans traînant par terre et évasés comme une corolle, de sorte qu'avec sa coiffure à coques et chignon imitée des estampes japonaises son visage un peu gras vierge de hâle on aurait dit quelque délicate tête de porcelaine blanche et noire surmontant un pavillon de phonographe posé à l'envers) ... au recto, donc [...].

Le procédé comparatif marque une sorte de mouvement de retrait du narrateur présentant le récit comme pure simulation. Pour Roland Barthes, le «comme si » est justement une simulation de la narration : une fiction théorique ou un rapport à la réalité qui s'institue comme fiction. De fait, la description ouvre sur un contenu qui, à la fois, s'avère crédible tout en suggérant l'illusion dont il est constitué ; cela rejoint une tendance du Roman dans son rapport à une certaine mythologie de l'universel, selon BARTHES (1953 : 50-51), dans Le Degré zéro de l'écriture :

[...] donner à l'imaginaire la caution formelle du réel, mais laisser à ce signe l'ambiguité d'un objet double, à la fois vraisemblable et faux [...] le faux égale le vrai, non par agnosticisme ou duplicité poétique, mais parce que le vrai est censé contenir un germe d'universel, ou si l'on préfère, une essence capable de féconder, par simple reproduction, des ordres différents par l'éloignement ou la fiction.

Tout indique dans le récit simonien qu'il est impossible de restituer fidèlement la réalité par une auto-analyse constante de l'énoncé dans toutes les suppositions qu'il induit face au caractère irrésolu des problématiques qui se révèlent au fur et à mesure du déroulement narratif, jusqu'à en laisser apparaître la profonde 
ambiguïté : tandis que l'énoncé cherche à renvoyer à la fiction, l'écriture est soumise à une réverbération signifiante qui prend acte continûment de ses propres imperfections. En tentant de dire ce qui n'a pas vraiment d'équivalent littéral, le narrateur contourne son impossibilité à transcrire ce qu'il est en train d'éprouver : il remplace la difficulté énonciative (comment puis-je décrire la réalité ?) par l'interrogation perceptive (comment est-ce que je la perçois ?). Cela se repère notamment lorsque, au lieu de répondre de la façon la plus claire à l'exigence informative de sa fonction en renvoyant à un fait d'expérience connu du lecteur, la description de la réalité sensible paraît empreinte de relativité, soumise au style prudent de celui qui ne se risque qu'à regret, trahi par des peut-être, des sans doute, comme si la perception des choses mêmes en avait déréglé le fonctionnement normal. Il en ressort que la description, dictée par l'émergence des choses mêmes, paraît chercher ses mots pour tenter de les prendre en charge. En ne cessant de repasser sur ses traits, pour les prolonger, l'écriture complexifie en permanence ce difficile rapport aux choses, où toute certitude s'ébranle face à l'accumulation d'images évocatoires strictement hypothétiques. Ainsi, dans cette mise en abyme descriptive qui la caractérise, la restitution entraîne à sa suite le lecteur dans la reconstitution de la scène. Soumise à une relance infinie, la description favorise en permanence l' aspect non conclusif de ce dont elle témoigne car elle ne semble jamais vouloir se refermer sur des frontières repérables. Dans la résonance sensible du texte, s'ouvre tout un espace de développements où il n'est pas tant question de clarifier la réalité que de la montrer comme insaisissable, en rendant complexes ses modalités d'appréhension. N'étant pas assentiment au monde tel qu'il est, le récit joue sur le caractère plausible de ce qu'il avance. De cette friction avec la réalité naît tantôt un ajout, tantôt une soustraction, mais jamais une jonction parfaite, de telle manière que le récit semble émerger de la rencontre improbable entre le dire et son objet d'attention, laissant apparaître que la rationalité de la narration pâtira tôt ou tard d'un tel rapprochement. En forgeant avec subtilité une pure fiction à partir de la réalité à laquelle il feint de vouloir renvoyer, le récit en aiguise tantôt la perception ou tout au contraire la dissimule à loisir sous un trop-plein d'informations. D'où l'impression que la réalité n'est finalement qu'une toile de fond, un simple décor sur lequel peut en toute liberté s'épanouir à sa guise la description, à la recherche constante de ses propres lois esthétiques forgées au détriment de toute logique. N'étant assujettie à aucun réalisme, la description n'a plus qu'à détourner la contrainte du vraisemblable à son profit pour se mettre au service du jeu avec la perception sans même chercher à produire une illusion référentielle. Par ces ruses du récit, il est question de la perpétuelle évolution des choses, éclairées par la description des sensations et de l'indicible qui les enveloppe. Aussi, par le caractère fragmentaire et chaotique des éléments qu'il donne en simultanéité, le récit métis donne l'illusion de ne pas pouvoir vraiment tenir les choses sous son ordonnance tant celles-ci, irréductibles à toute description, la neutralisent. C'est ainsi que la narration, comme indifférente à la rigueur du réel, annule son emprise en ménageant un dégagement, une voie libre pour l'invention : le simulacre du récit, qui plaide pour le caractère immanent du langage, produit une sorte d'effet de réel. 


\section{L'effet de réel}

Dans Histoire, l'irrésistible attrait du narrateur pour l'image peut tout aussi bien qualifier la fascination qu'elle exerce sur Claude Simon, en raison du statut particulier qu'il lui donne: idéalité en un sens, elle est ouverture ontologique. Ainsi la réflexion sur l'image réverbère un savoir sur la nature des choses et des êtres : dans la perception, un savoir se forme lentement, si tant est que l'on puisse le saisir pour soi-même et le restituer dans une écriture de la sensation. Cela caractérise la narration qui s'attarde longuement sur la photo d'un atelier de peinture. Après avoir transité par différents prismes entre éloignement et focalisation, la photo se précise à la conscience du narrateur jusqu'à se cristalliser en lui comme le ferait une image mentale. Cela s'indique lorsque la narration, se déplaçant d'un point de vue extérieur, objectivé, pénètre dans la scène pour la décrire; par ce processus d'intériorisation, elle devient de plus en plus introspective afin de développer l'image fixe en récit. La description, libérée des contraintes du réel, s'autorise à imaginer les moments précédant la prise de vue et ceux qui l'ont suivie. C'est une façon de ranimer ces tranches de vie condensées dans l'image, de relancer le temps suspendu par le contact photographique. L'aura de l'image conserve une trace vive de tout ce qui, sans elle, aurait pu être oublié, et témoigne de quelque chose qu'il est impossible de réduire au silence (BENJAMIN, 2000 : 300). Voilà pourquoi l'auteur l'utilise comme un révélateur, dans la rigueur du terme, qui lui permet d'imaginer à partir d'un état de latence tout ce que le visible peut receler en lui-même. L'association visuelle du narrateur à l'image opère une médiation privilégiée entre le sujet et le monde, le percevant et le perçu, qui agit comme un entrelacs, un chiasma :

Le chiasma n'est pas seulement moi autrui [...] c'est aussi échange de moi et du monde, du corps phénoménal et du corps « objectif », du percevant et du perçu : ce qui commence comme chose finit comme conscience de la chose, ce qui commence comme « état de conscience » finit comme chose. (MERLEAU-PONTY, $1964: 268$ )

En se concentrant sur la trame qui relie tous les personnages de la photo patiemment exploitée dans toutes ses possibilités, la description dépeint par excès ou en creux, mais jamais totalement en phase avec le modèle : ni description réaliste ni même réalisme dans la description. Sans contrat référentiel ni projet d'exécution repérable, il n'existe aucune tension narrative explicite qui exige l'élection de tel ou tel motif, mis à part un constant va-et-vient entre la scène et le passé qui l'a précédée. Cette impression est renforcée par un brusque changement d'identité qui s'opère chez le narrateur: un échange de pronom personnel intervient inopinément, ce qui accentue davantage la confusion ressentie jusqu'alors. Le « il » de l'oncle Charles devient sans raison apparente le « je » du neveu (le narrateur), alors que rien n'indique dans le récit un changement de régime ou une transformation nécessaire. Dans ce brouillage de référentialité, le passage au « je » désigne l'implication irrépressible du narrateur (le neveu) dans la scène, d'où la confusion qui s'ensuit dans les pronoms : à force de scruter la scène et de la décrire avec grande minutie, il se substitue à l'oncle Charles pour devenir un protagoniste, engagé à son tour dans les méandres du récit. Un élément, 
introduit incidemment, peut expliquer un possible lien de causalité : on apprend au cœur d'une phrase sinueuse à l'envi que le neveu s'intéresse à la nature des relations qui unissent son oncle et la jeune femme à moitié nue qui sert de modèle au peintre car ils sont, à ses yeux, les acteurs principaux de la scène. L'échange dans les pronoms marque ainsi une substitution des rôles qui relève d'un désir mimétique, où se marquent des effets de convoitise plus ou moins inconscients chez celui qui, rival du désir modèle qu'il cherche à imiter, se sent frustré d'une possession qu'il estime devoir lui revenir. C'est là une façon de désirer intensément une chose dont il se sent privé et dont quelqu'un d'autre lui paraît pourvu : «[...] le désir est essentiellement mimétique; il se calque sur un désir modèle ; il élit le même objet que ce modèle » (GIRARD, 1972 : 217). Animé d'un tel désir, qui l'a conduit à usurper la place qu'il convoitait, le neveu est passé d'une observation générale, distante, à la découpe minutieuse et attentive de la scène analysée et scrutée dans ses moindres détails au fur et à mesure qu'il s'impliquait davantage, jusqu'à endosser le rôle de son personnage par une forme d'hallucination visuelle. Ainsi, la confusion dans l'échange impromptu des pronoms personnels peut-elle se mesurer à l'aune du puissant vertige ressenti par le narrateur, qui en scrutant l'image espère trouver dans cette forme d'incarnation généalogique des éléments de la vie supposée de son oncle (DÄLLENBACH, 1988 : 57) :

[...] ce qui commande le regard du sujet, c'est la révélation qu'il escompte du seul « document » qu'il possède sur la vie de Charles à Paris ; [...] qui pourrait bien être la naissance d'un drame (la liaison de Charles avec le modèle marquant le début de la désunion de son mariage et apparaissant comme la cause de la mort de sa femme) - d'un drame (nous le savons par des indices furtifs) analogue au sien (à une génération près), les deux destinées se constituant et s'interprétant l'une par l'autre.

Informulé, le suicide d'Hélène (femme du narrateur), est le drame suggéré par tout un réseau de significations qui le désigne comme centre évidé du roman, une histoire déjà là (SARKONAK, 1986: 23). Et si la photo est perçue comme une archive biographique, alors le transfert d'identité est peut-être motivé par des causes psychologiques car le moment clé de la perception des taches est décrit sans relation de causalité logique avec quelque événement qui puisse l'expliquer autrement que par une telle approche (DÄLLENBACH, 1988 : 59) :

[...] faut-il s'étonner que ce qui détermine le transfert du neveu sur l'oncle... soit la perception soudaine des taches de peinture qui tout à la fois réactivent les motifs qui courent à travers tout le roman et unissent oncle et neveu en les logeant à la même enseigne : celle d'une sexualité fatale sanctionnée par un sentiment de culpabilité et de souillure ?

Le transfert d'identité fait irruption dans le temps du récit pour interrompre toute liaison référentielle et produire un effet de brouillage, dont l'activation vise à reproduire la situation réelle du voir quand les choses cessent de correspondre aux représentations permettant de les reconnaître. Dans la description, l'apparition des taches fait suite au déplacement irrationnel du regard qui parcourt sans but précis la scène au travers de filtres successifs réduisant les objets au bon vouloir d'une perception libre : 
[...] le quadrillage de la chemise écossaise à travers lequel on pouvait voir, comme derrière une grille, l'occupant du fauteuil d'osier qui a posé maintenant sa tasse sur un tabouret penché à son tour en avant, les avant-bras appuyés sur les cuisses écartées, les deux mains jointes dont l'une tient la blague à tabac et l'autre la pipe qu'il est en train de bourrer tandis qu'à ce moment [ici, l'auteur a procédé à une interruption inopinée dans le texte, qui se manifeste par un saut à la ligne]. / il en tomba quelques-uns jaune-blond frisés entre mes pieds je vis alors que le plancher était maculé de taches. Couleur tombée du pinceau trop chargé. Constellation. (SimON, 1967 : 289- 290)

Par une telle rupture, le récit délaisse toute logique au profit de la sédimentation des sensations visuelles: l'apparition insolite du groupe de mots «quelques-uns » ouvre une perception qui se révèle en simultanéité. Tout incite à imaginer, sans qu'il en soit fait mention, que des brins de tabac, en chutant au sol, ont entrainé par attraction visuelle le regard du narrateur sur les taches de peinture. Provoqué par le transfert des identités, le mouvement d'intériorisation intervient de manière improbable, comme si le narrateur épousait son propre point de vue dans une fusion du regard et de l'objet de son élection. Mais la confusion des identités, par laquelle le neveu (le narrateur) se substitue inopinément à son oncle, peut trouver une explication s'écartant de tout rapport psychologique : le chiasme visuel, convergence du temps et de la perception, fait remonter l'image d'un temps inactuel, l'exhume comme un fossile ramené du fond des mondes imaginaire. En effet, pour MERLEAU-PONTY, le visible n'est pas un morceau d'être, insécable, mais une sorte de " détroit entre des horizons extérieurs et des horizons intérieurs toujours béants »(1964:175). Ici, dans la potentialité qu'il contient, et qu'il s'agit d'activer par des modalités littéraires appropriées, le visible est prétexte à toutes les élucubrations. En prenant appui sur une photo, comme structure perceptive de l'Image, le narrateur mène sa propre enquête. Au sujet de Histoire, l'auteur précise sa démarche : «En grec ancien le mot istoria ne signifie pas "histoire" dans le sens où nous l'entendons aujourd'hui, mais recherche, enquête. C'est cela qui m'intéresse, cette recherche de ce que l'écriture va m'apporter» (KNAPP, 1969: 189). Ces mots pourraient s'appliquer à l'intention du narrateur, dont la quête minutieuse semble vouloir pénétrer la sphère de l'image pour sonder le noème qui lui est propre, le type spécifique d'apparaître conservé par-devers elle, et restituer l'origine d'où elle provient (BENJAMIN, 1985 : 43-44) :

L'origine ne se donne jamais à connaître dans l'existence nue évidente du factuel, et sa rythmique ne peut être perçue que dans une double optique. Elle demande à être reconnue d'une part comme une restauration, une restitution, d'autre part comme quelque chose qui est par là même inachevé, toujours ouvert.

Ignorant presque tout de l'événement, le lecteur est conduit à en restaurer par lui-même les manques ou, à tout le moins, à lever les incertitudes qui lui restent attachées en procédant à divers recoupements pour faire surgir ce qui, en son fond, palpite comme un vestige-témoin. Construit, tout autant que déconstruit, le récit se présente comme une simple postulation soumise à l'appréciation du lecteur, libre de produire ses propres interférences sur l'événement fixé par cette dialectique à l'arrêt : 
Il ne faut pas dire que le passé éclaire le présent ou que le présent éclaire le passé. Une image, au contraire, est ce en quoi l'Autrefois rencontre le Maintenant dans un éclair pour former une constellation. En d'autres termes : l'image est la dialectique à l'arrêt. (BENJAMin, 1989, 479480)

C'est donc bien le mouvement antérieur qui a précédé le temps de la pose, dont la scène n'est que le point d'aboutissement, que la description cherche à restituer; fournie, elle semble vouloir remonter le temps en retraçant le déroulement complet de la prise de vue qui témoigne d'un ça a été (BARTHES, 1980 : 124). Or, si cette archive offre une voie d'accès privilégiée à une biographie familiale, elle comporte aussi une part d'inconnu qu'il importe de réduire; conjecturale, elle renferme un pan du passé qui autorise toutes les suppositions imaginables, tant il n'est possible d'en retracer qu'une trame purement hypothétique. Pour tout un chacun, le temps archivé dans des photos de famille se retrouve à l'état de survivances imaginaires. Le plus souvent sa trame est constituée du récit que d'autres en font, réduisant chaque événement à une scorie sur laquelle la réalité se bâtit, par glissements successifs, de récits en photos ; imaginaire, ce temps rapporté informe par ouï-dire et constitue des souvenirs d'emprunts sans trace mnésique. D'où l'intérêt pour le narrateur de scruter la photo avec la plus grande application pour sonder le mystère qu'elle abrite et pallier la connaissance forcément lacunaire de la mémoire. Si l'épreuve photographique a figé une empreinte de temps, comme un suaire, la description cherche à honorer les êtres présents, à ranimer ces moments de vie révolus, par une exploration minutieuse qui, au-delà des perceptions immédiates, s'attaque à l'énigme afin d'en restituer les informations enfouies. L'image devient alors un support privilégié de rêverie à partir duquel l'imaginaire tente de reconstituer, fragment après fragment et par retouches successives, le moment de vie qu'elle a emprisonné. Ainsi la description exploite-t-elle l'image comme un humus où ont pu croître et se modifier des souvenirs, en raison de l'attraction irrésolue qu'elle exerce par son pouvoir de fascination. Mais qu'elle choisisse de contrarier la perception en dissimulant sous l'abondance de détails cela même qu'elle s'efforce de traduire ou qu'elle feigne de la rendre plus claire, elle rend incertaine la réalité qu'elle transcrit. Malgré sa densité, elle n'arrête aucune sentence et se trouve sans cesse relancée par les multiples associations qu'elle suscite; en résistant à la compréhension logique, elle oblige le lecteur à établir des liens entre tous les constituants du texte.

Tout cela est renforcé par la plurivocité et la mutabilité de la signification induite par l'hésitation de l'écriture qui, en cherchant ses mots pour s'adapter au contexte, semble livrée au pouvoir créatif d'une langue naturelle :

La variabilité des significations, en particulier les déplacements de sens nombreux [...] sont précisément les propriétés qui favorisent la créativité d'une langue naturelle [...]. Ici, l'indéterminé et le pouvoir créateur apparaissent comme totalement solidaires. (JAKOBSON, $1970: 508)$

Lorsque l'auteur incorpore des mots au développement puis feint de les rejeter après les avoir testés à plusieurs reprises, il confère à la constitution même du récit 
des possibilités d'invention continues par des associations métonymiques permanentes par groupes de substitution (JAKOBSON, 1963 : 48-49). Si la photo a pu capter la part d'imprévu qui entre dans l'existence, il convient alors de ranimer la scène qu'elle a figée. Mais les comportements humains ne pouvant pas toujours se réduire à des règles identifiables, le récit doit tenter de rendre compte des choses dans l'ordre de l'expérience vécue. $\mathrm{Si}$, pour Claude Simon, l'image abrite un «mouvement immobile », encore faut-il en relancer la dynamique figée dans l'empreinte photographique. Ouverte sur d'incessantes bifurcations métaphoriques, la narration développe autant d'ambiguités sémiotiques qui ont pour effet de confondre le monde avec son image, le factuel avec l'illusion, dans un maniement subtil de l'hypotypose (description animée), figure de rhétorique qui consiste à susciter l'imagination pour animer les choses décrites. La photo apparaît alors comme un objet qui n'existe pas autrement qu'en tant qu'image de lui-même : un objet virtuel en mutation permanente qu'il s'agit de décrire dans une représentation insolite (CHKLOVSKY, 1973: 17). Cela se repère quand la description ne mentionne pas des traits de l'objet permettant de le reconnaitre, pour favoriser des détails généralement négligés, inutiles, car sans réelle pertinence par rapport à sa fonction. Par une telle volonté, qui en dénature l'approche fonctionnelle jusqu'à le rendre insolite, l'objet perd sa présence habituelle. Comme si la restitution du réel devait entretenir un autre rapport aux choses sans se préoccuper d'être fidèle à quelque vérité pour en traduire une nouvelle perception. Pour MERLEAU-PONTY il faut voir les choses non pas négligemment, «du coin de l'œil», soumises à un regard accoutumé, mais les observer avec une acuité analytique pour en révéler toute l'étrangeté :

Nous ne nous en avisons pas d'ordinaire parce que notre perception, dans le contexte de nos occupations, se pose sur les choses juste assez pour retrouver leur présence familière et pas assez pour redécouvrir ce qui s'y cache d'inhumain. (MERLEAU-PonTY, 1945 : 372)

Ce procédé, qui consiste à débarrasser la vision de ses automatismes pour susciter un sentiment d'étrangeté, se retrouve dans de longues phrases, propres au style simonien, sans début ni fin, qui décrivent les choses selon un mode différent de leur perception classique. Reposant sur une narration libre et inventive, l'extrême attention portée au moindre détail s'évertue à produire une certaine illusion de réalité, un effet de réel, pour montrer la nature propre des choses du monde, comme indépendante de nos perceptions et de notre désir de logique. Aussi, le flux de déductions pour imaginer les moments précédant la prise de vue et les finesses d'observation pour analyser la photo apparentent le récit à une enquête active, sans déroulement narratif linéaire ni fil conducteur clairement identifiable. Dans son errance, le récit ouvre des espaces segmentés en sentiers inconnus, mêlant les choses réelles et celles concédées au fantasme pour entraîner le lecteur à sa suite par sa puissance suggestive. Cela donne l'impression que la photo a été ranimée par une prosopopée de la description, à laquelle l'auteur associe l'usage métaphorique qui permet à la forme narrative de se dégager du réel en faisant endosser un sens figuré à l'objet décrit, différent de celui qui, ordinairement, l'habite. Mais la description se complexifie encore d'un degré du 
fait même que la métaphore n'est pas appliquée à l'objet, mais y est, dès l'origine, conjointe. Elle ne vient pas, comme de coutume, en appui de l'objet dénoté ; elle s'inscrit à l'intérieur de celui-ci, pour lui insuffler la vie et, par contagion signifiante, en modeler des contours neufs. Le procédé caractérise, entre autres aspects singuliers, l'écriture simonienne qui cherche à conquérir sa propre autonomie sur la réalité décrite. Voilà pourquoi, dans Histoire, la description de la photo, malgré sa ferme volonté de ne rien oublier, semble animée d'un mouvement propre qui l'éloigne du monde qu'elle traite; par une absence de caractère effectif, elle peut côtoyer l'irreprésentable, ce qui, passant au travers des mailles du réel, n'a pu être capturé par l'image.

\section{Conclusion}

Le texte simonien honore la contingence du perçu en impliquant le lecteur grâce à de constantes allusions qui, adjacentes au développement, permettent d'y associer des possibilités qui le prolongent. De sorte que la narration se déroule comme si toutes les perceptions sensorielles, au lieu de rester intérieures étaient projetées au-dehors, et demandaient à être partagées pour la mise en forme cohérente du réel. En mettant l'accent sur une mise en commun de l'appréhension des choses, celles-ci sont incorporées au déroulement descriptif par l'effet d'une projection qui donne corps à la réalité perçue par les sens et par la conscience. Paradoxal est ce regard à la fois réglé sur la scène décrite et sur ce qui l'en déporte par abduction, au point parfois de ne laisser paraître qu'une intention sans objet autre que celle de montrer l'énigme des choses considérées sous une double optique: l'acuité d'un regard et une indifférence fascinée. La description dynamique ne se contente pas de reproduire le réel mais le produit au travers de l'«objet écrit»; pour cela, elle s'impose certains effets de contrainte par l'utilisation des peut-être, ou plutôt, à moins que, où se marque un ajustement permanent de l'énoncé à l'objet. Comme si le texte s'adaptait aux choses décrites dans une tentative de synchronisation à postériori, par un procédé cherchant à reproduire ce qu'il en est de l'éclatement des impressions et des sensations. C'est pourquoi, durant de longues plages descriptives, le développement transite par plusieurs effets successifs (ou simultanés) : tantôt la narration feint de s'approcher de la réalité, cernée au plus près par de nombreux détails pointillistes, tantôt elle cherche à s'en dégager par le biais de touches descriptives vaporeuses, quasi abstraites même. Cette spécificité de la description, en débordant le cadre de la scène qu'elle s'impose, exprime toute sa duplicité : elle se donne pour le reflet imposé et informé par un réel préexistant ou possible et elle suggère que ne s'y trouve pas en fait ce qu'il y apparaît. Tel est le pouvoir d'évocation de cette écriture, qui permet de rebondir sur la matérialité de l'objet, allégué par le recours à des sensations qui en étirent les contours; mais elle est aussi dotée d'une fonction mnésique qui ramène à la mémoire des survivances du passé et à la conscience des souvenirs enfouis, des réminiscences. Dynamique, l'écriture simonienne est à la fois une figure d'appel et de rappel, où les choses semblent être perçues comme des images mentales. Pour Sartre, les choses sont différentes de la description que l'on peut en donner, car dans les opérations de la conscience 
imageante, l'image mentale produit une illusion détachée de la perception réelle, où l'objet n'est que la projection de ce que l'observateur voit. En produisant une illusion, qui la fait passer pour quelque chose de consistant dans la conscience, l'image mentale nous fait croire à du donné quand elle n'est que du posé, comme une fiction (SARTRE, 1936). Quand elle cherche à saisir l'essence de l'objet perçu, une illusion similaire nourrit le flux descriptif par l'accumulation d'innombrables détails superflus essaimés comme autant d'éléments fertilisants - la nature des choses, ne pouvant être saisie avec certitude, s'offre comme éminemment hypothétique (d'où l'usage fréquent des formules du style «il me semblait le voir »). Telle apparaît la ruse du récit, qui élabore un monde et, simultanément, feint de le découvrir dans la construction même du texte. C'est une façon habile d'utiliser la réalité comme le sujet, le point départ propice à des commentaires infinis (prédicat) tout en traitant l'écriture comme l'objet de référence ultime, le point d'arrivée vers quoi converge sa conception des choses. Aussi l'impression subsiste qu'il n'est pas tant question d'accéder au monde réel que de simuler le fonctionnement du langage par l'usage singulier de la description, où sans cesse l'intention du narrateur se confond avec l'orientation objective du récit.

\section{BIBLIOGRAPHIE}

BARTHES Roland (1980), La chambre claire, Note sur la photographie, Paris, Seuil.

BARTHES Roland (1953), Le Degré zéro de l'écriture, Paris, Seuil.

BENJAMIN Walter (1985), Origine du drame baroque allemand, Paris, Flammarion, traduit de l'allemand par Sibylle MULLER.

BeNJAMIN Walter (1989), Paris, Capitale du XIX ${ }^{\text {ème }}$ siècle. Le livre des passages, Paris, Cerf, traduit de l'allemand par Jean LACOSTE.

BENJAMIN Walter (2000), Petite histoire de la photographie, in : Euvres, II, Paris, Gallimard, coll. "Folio-Essais », traduit de l'allemand par Maurice de GANDILLAC, Rainer RochlitZ et Pierre RusCH.

ChKLOVSKY Victor (1973), Sur la théorie de la prose, Lausanne, L'âge d'homme, coll. «Slavica », traduit du russe par Guy VERRET.

DÄllenbaCH Lucien (1988), Claude Simon, Paris, Seuil, coll. «Les contemporains ».

GIRARD René (1972), La violence et le sacré, Paris, Grasset.

JAKOBSON Roman (1970), La Linguistique, in: Tendances principales de la recherche dans les sciences sociales et humaines, Paris-La-Haye, MoutonUnesco.

JAKOBSON Roman (1963), Essais de linguistique générale, Paris, Minuit.

JANVIER Ludovic (1964), Une parole exigeante. Le nouveau roman, Paris, Minuit.

KNAPP Bettina L. (1969), Interview avec Claude SIMON, in : Kentucky Romance Quarterly, vol. 16, n 2, p. 189.

MERLEAU-PONTY Maurice (1964), Le visible et l'invisible, Paris, Gallimard, p. 268. 
Merleau-Ponty Maurice (1945), Phénoménologie de la perception, Paris, Gallimard, p. 372.

POIRSON Alain ; GouX Jean-Paul (1977), Un homme traversé par le travail, in : La nouvelle critique, $\mathrm{n}^{\circ} 105$, juin-juillet, p. 32-44.

RICARDOU Jean (1978), Le dispositif osiriaque, in: Nouveaux problèmes du roman, Paris, Seuil, coll. « Poétique », p. 185-186.

SARKONAK Ralf (1986), Claude SIMON: les carrefours du texte, Toronto, Paratexte.

SARTRE Jean-Paul (1936), L'imagination, Paris, PUF.

SIMON Claude (1967), Histoire, Paris, Minuit.

VAREILLE Jean-Claude (1989), À propos de Claude SiMON : langage du cosmos, cosmos du langage, in: Fragments d'un imaginaire contemporain, PINGET, RoBbE-GRILLET, SIMON, Paris, José CORTI, p. 90. 